\title{
Resveratrol pretreatment attenuates traumatic brain injury in rats by suppressing NLRP3 inflammasome activation via SIRT1
}

\author{
PENG ZOU ${ }^{1 *}$, XIAOXIAO LIU ${ }^{2 *},{\text { GANG } \mathrm{LI}^{3} \text { and YANGANG WANG }}^{1}$ \\ ${ }^{1}$ Department of Neurosurgery, Xijing Hospital, Fourth Military Medical University; \\ ${ }^{2}$ Department of Cardiology, Xi'an Medical University Affiliated Northern Hospital, Xi'an, Shaanxi 710032; \\ ${ }^{3}$ Department of Neurosurgery, Jiangxian People's Hospital, Yuncheng, Shanxi 043600, P.R. China
}

Received June 20, 2017; Accepted November 21, 2017

DOI: $10.3892 / \mathrm{mmr} .2017 .8241$

\begin{abstract}
The inflammatory response in the cerebral cortex serves an important role in the progression of secondary injury following traumatic brain injury (TBI). The NLR family pyrin domain containing 3 (NLRP3) inflammasome is necessary for initiating inflammation and is involved in various central nervous system disorders. The aim of the present study was to investigate the neuroprotective effect of resveratrol and elucidate the underlying mechanisms of resveratrol associated regulation of the NLRP3 inflammasome in TBI. The results demonstrated that the activation of NLRP3, caspase-1 and sirtuin 1 (SIRT1), enhanced the production of inflammatory cytokines and reactive oxygen species (ROS) following TBI. Administration of resveratrol alleviated the degree of TBI, as evidenced by the reduced neuron-specific enolase (NSE) and brain water content (WBC). Resveratrol pretreatment also inhibited the activation of NLRP3 and caspase-1, and reduced the production of inflammatory cytokines and ROS. In addition, resveratrol further promoted SIRT1 activation. Furthermore, the suppressing effect of resveratrol on the NLRP3 inflammasome and ROS was blocked by the SIRT1 inhibitor, sirtinol. The results revealed that the activation of the NLRP3 inflammasome and the subsequent inflammatory responses in the cerebral cortex were involved in the process of TBI. Resveratrol may attenuate the inflammatory response and relieve TBI by reducing ROS production and inhibiting NLRP3 activation. The effect of resveratrol on NLRP3 inflammasome and ROS may also be SIRT1 dependent.
\end{abstract}

Correspondence to: Dr Yangang Wang, Department of Neurosurgery, Xijing Hospital, Fourth Military Medical University, 127 Changle West Road, Xi'an, Shaanxi 710032, P.R. China

E-mail: yg-wang@163.com

*Contributed equally

Key words: traumatic brain injury, resveratrol, NLR family pyrin domain containing 3, inflammasome, reactive oxygen species, sirtuin 1

\section{Introduction}

Traumatic brain injury (TBI) is a common severe injury of neurosurgery, which is mainly caused by mechanical forces of tearing, shearing or stretching. TBI has been a heavy economic and social burden and has high morbidity and mortality (1). So far plenty of researches have been conducted to illuminate the mechanisms underlying TBI and explored the effective neuroprotective drugs aiming to alleviate the grave consequences of TBI. Although the precise mechanisms underlying the development of TBI remain unclear, it is admitted that the secondary injury is tightly linked with the prognosis of TBI. Post-traumatic inflammation response in cerebral cortex plays an important role in the progression of secondary injury (2).

Increasing evidences indicate that cytokines, such as IL- $1 \beta$, participate in inflammation development. IL- $1 \beta$ is a potent inflammatory initiating cytokine. It can activate additional inflammatory cells and release more inflammatory mediators and cytokines, thus amplifying the injury signals and triggering an inflammatory cascade (3). IL-1 $\beta$ is mainly produced by NLRP3 inflammasome, which serves as a platform to activate caspase and triggers proteolytic maturation of pro-inflammatory cytokines, such as IL-1 $\beta$ and IL-18 (4). Increasing evidences indicate that NLRP3 inflammasome participates in the development of central nervous system disorders, such as cerebral ischemia reperfusion injury (5), neurodegenerative disease (6), cerebral tumor (7) and so on. It is reported that NLRP3 also participates in the pathogenesis of TBI (8).

Resveratrol (3,5,4'-trihydroxy-stilbene, RS) is a stilbenoid, a natural polyphenolic agent, which has been demonstrated to eliminate free radicals and ROS, reduce inflammatory response and modulate programmed cell death (9). Particularly, administration of resveratrol has potent neuroprotection effects in various animal models including cerebral ischemia reperfusion injury (10), stroke (11), and TBI (12). However, the mechanisms through which resveratrol exerts protective effect following TBI require further examination. A previous study demonstrated resveratrol exerted a protective effect in acute lung injury by suppressing the NLRP3 inflammasome signaling (13). So it is necessary to determine whether resveratrol is capable of modulating TBI through NLRP3 inflammasome signaling pathway. 
In the present study, we sought to investigate the neuroprotective effect of resveratrol and to explore the underlying mechanisms of resveratrol regulating NLRP3 inflammasome in TBI.

\section{Materials and methods}

Animals and experimental groups. Healthy adult male Sprague-Dawley rats $(300 \mathrm{~g}-330 \mathrm{~g})$ were used in the experiments, which were purchased from the Experimental Animal Center of the Fourth Military Medical University. The present study was approved by the Ethics Committee of Animal Experiment Administration Committee of The Fourth Military Medical University (Shaanxi, China). Rats were housed under humidity and temperature controlled conditions as well as under specific pathogen-free conditions. Rats were randomly divided into the following groups: sham group $(n=6)$, TBI group at different time point $(n=24), T B I+R e s v e r a t r o l(R S)$ group $(n=6)$ and TBI+sirtinol group $(n=6)$.

TBI model. The detailed methods to produce TBI model had been described previously (1). Briefly, we used a free fall device in the experiment. Rats were anaesthetized using pentobarbital sodium $(50 \mathrm{mg} / \mathrm{kg})$ by intraperitoneal injection and then a midline incision was created, and the skin was retracted to expose sagittal suture and coronal suture. When fixed to the free fall device, a ball breaker weighting $50 \mathrm{~g}$ was dropped from the $90 \mathrm{~cm}$ height to form a free-falling impact, resulting in closed craniocerebral trauma. Almost all animals subjected to TBI showed brain hemorrhage and edema, however, generally $10 \%$ mortality post injury was observed.

Resveratrol (Sigma, St Louis, MO, USA) was dissolved in $75 \%$ ethanol and attenuated by normal saline (2\% ethanol content) to a concentration of $100 \mathrm{mg} / \mathrm{ml}$. $100 \mathrm{mg} / \mathrm{kg}$ resveratrol was administered intraperitoneally in the TBI+RS group $30 \mathrm{~min}$ before TBI (14), while a corresponding volume of saline ( $2 \%$ ethanol content) was administered in the TBI group and sham group $30 \mathrm{~min}$ before TBI model. The sham group rats were subjected to an identical procedure with the TBI group rats, however without ball breaker hit.

Sirtinol (Selleck Chemicals, Houston, TX, USA), a potent SIRT1 inhibitor, was dissolved in $75 \%$ ethanol and normal saline ( $2 \%$ ethanol content). Sirtinol $(10 \mathrm{mg} / \mathrm{kg})$ was administered intraperitoneally $30 \mathrm{~min}$ before TBI in the TBI+sirtinol group (15).

In the TBI group, rats were sacrificed at 6, 12, 24 and $48 \mathrm{~h}$ after TBI respectively, and their tissues were sampled. In the other groups, rats were sacrificed, and their tissues and blood were sampled $24 \mathrm{~h}$ after TBI. Blood was taken from aortaventralis and centrifuged at 1,500 $\mathrm{g}$ for $10 \mathrm{~min}$ to collect serum.

Enzyme-linked immune sorbent assay (ELISA). The serum level of neuron-specific enolase (NSE), a specific biomarker of brain injury, was quantified using corresponding ELISA kit (Jiancheng Bioengineering Institute, Nanjing, China). The level of IL-1 $\beta$ and IL-18 in the supernatant of cerebral cortex homogenate was determined using specific ELISA kit (Jiancheng Bioengineering Institute) according to the manufactures' protocols. The optical density (OD) values were measured at a wavelength of $450 \mathrm{~nm}$ using ELX808 microplate reader.

Measurement of brain water content $(B W C)$. Brain water content was measured by wet/dry weight ratio according to the protocol described previously (16). In brief, the injured cerebral hemisphere was weighted and then transferred to desiccating oven for dehydration at $110^{\circ} \mathrm{C}$ for $24 \mathrm{~h}$. The dried tissue was weighted again. $\mathrm{BWC}=[$ (wet brain weight-dry brain weight)/wet brain weight]x $100 \%$.

Measurement of the level of SOD, MDA and GSH in rats cerebral cortex. The level of SOD, MDA and GSH were spectrophotometrically assayed using appropriate kits (Jiancheng Bioengineering Institute) respectively. The cerebral cortex was homogenized and centrifuged in extraction buffer to obtain a 5\% homogenate. The absorbance was measured using a DU-800 spectrophotometer (Beckman Coulter, Fullerton, CA, USA) at a wavelength of $460 \mathrm{~nm}$.

Quantitative real-time reverse transcription PCR ( $q R T-P C R)$. The mRNA levels of NLRP3, caspase-1, IL-1 $\beta$ and IL-18 were analyzed by qRT-PCR. We used TRIzol (Invitrogen Life Technologies, Carlsbad, CA, USA) to extract total RNA in injured cerebral cortex. RNA was reverse-transcribed into cDNA by Prime Script ${ }^{\mathrm{RT}}$ Reagent kit (Takara Bio, Inc., Otsu, Japan). Using SYBR Green (Takara Bio, Inc.) and specific primers, the levels of target gene mRNAs were measured with a real-time PCR system (Bio-Rad Laboratories, Inc., Hercules, CA, USA).

The qRT-PCR thermalcycle condition was employed as follows: an initial denaturation at $95^{\circ} \mathrm{C}$ for $30 \mathrm{sec}$, followed by 39 cycles of denaturation at $95^{\circ} \mathrm{C}$ for $15 \mathrm{sec}$, annealing at $60^{\circ} \mathrm{C}$ for $30 \mathrm{sec}$, and elongation at $72^{\circ} \mathrm{C}$ for $10 \mathrm{sec}$.

The primer sequences of targeted genes used in the present study were as follows: NLRP3: 5'-CAGCGATCAACAGGC GAGAC-3' (F), 5'-AGAGATATCCCAGCAAACCTATCC A-3' (R); caspase-1: 5'-ACTCGTACACGTCTTGCCCTCA-3' (F), 5'-CTGGGCAGGCAGCAAATTC-3' (R); IL-1ß: 5'-CCC TGAACTCAACTGTGAAATAGCA-3' (F), 5'-CCCAAG TCAAGGGCTTGGAA-3' (R); IL-18: 5'-GACTGGCTGTGA CCCTATCTGTGA-3' (F), 5'-TTGTGTCCTGGCACACGT TTC-3' (R); GAPDH: 5'-GAACATCATCCCTGCATCCA-3' (F), 5'-CCAGTGAGCTTCCCGTTCA-3' (R).

Western blot. The injured cerebral cortex samples were homogenized and the concentrations of protein were measured using BCA assay method. $30 \mu \mathrm{g}$ protein was separated by electrophoresis on $10 \%$ sodium dodecyl sulfate-polyacrylamide gels, and transferred to polyvinylidene difluoride membranes. The following primary antibodies were used: NLRP3 (Biorbyt, Cambridge, UK), caspase-1 (Santa Cruz Biotechnology, Inc., Dallas, TX, USA) and SIRT1 (Abcam, Cambridge, UK). The sheared bands were observed by chemiluminescense using the FluorChem FC system and were presented as a densitometric ratio between the protein of target and the loading control. Results were analyzed using the software of ImageJ v1.46.

Statistical analysis. The experiment data were expressed as mean \pm SEM. Comparison between different groups was 
determined using one-way analysis of variance (ANOVA) followed by Bonferroni t-test using GraphPad Prism 6 software (San Diego, CA, USA). P $<0.05$ was considered to indicate a statistically significant difference.

\section{Results}

TBI resulted in elevated NLRP3 and caspase-1 expression in rats cerebral cortex. The expression of NLRP3 and caspase-1 at different time points after TBI were evaluated by western blot. Results indicated that the expression of NLRP3 and caspase- 1 were up-regulated at $6 \mathrm{~h}$ after TBI and peaked at $24 \mathrm{~h}(\mathrm{P}<0.05$; Fig. 1). So we chose the $24 \mathrm{~h}$ time point after TBI in our following experiments.

Resveratrol pretreatment reduced the $m R N A$ and protein expression level of NLRP 3 and caspase-1 in rats cerebral cortex after TBI. The mRNA expression of NLRP3 and caspase-1 were detected by real time PCR. The Fig. 2A and B showed that the mRNA expression level of NLRP3 and caspase-1 in the TBI group was significantly elevated compared with the sham group $(\mathrm{P}<0.05)$. Pretreatment with resveratrol remarkably reduced the elevated NLRP3 and caspase-1 mRNA levels $(\mathrm{P}<0.05)$. To determine whether the protein levels of NLRP3 inflammasome were corresponded to their mRNA levels, we further conducted western blot experiment. We observed the NLRP3 and caspase-1 protein level were increased after TBI $(\mathrm{P}<0.05)$, but significantly decreased by administration of resveratrol $(\mathrm{P}<0.05)$ (Fig. $2 \mathrm{C}$ and $\mathrm{D})$.

Resveratrol pretreatment decreased the levels of $I L-1 \beta$ and IL-18 in rats cerebral cortex after TBI. IL-1 $\beta$ and IL-18 are effector molecules of NLRP3 inflammasome signals, acting as inflammatory initiating cytokines. Therefore we analyzed the generation of IL-1 $\beta$ and IL-18 in rats cerebral cortex by real time PCR and ELISA. We found that the mRNA and protein level of IL-1 $\beta$ and IL-18 were relatively low in the sham group. TBI significantly increased the mRNA and protein level of IL-1 $\beta$ and IL-18 $(\mathrm{P}<0.05)$, while resveratrol pretreatment markedly decreased the elevated level of IL-1 $\beta$ and IL-18 $(\mathrm{P}<0.05)$ (Fig. 3A-D).

Resveratrol pretreatment decreased the level of serum NSE and brain edema in TBI rats. It has been demonstrated that resveratrol has the neuroprotective effect. First, we measured the serum level of NSE, a biomarker valuing brain injury degree. We found that NSE was significantly increased in serum of TBI rats. When pretreatment with resveratrol, the elevated NSE was remarkably abolished $(\mathrm{P}<0.05)$ (Fig. 4A). Moreover, the BWC in the TBI group was much higher than the sham group, which was reversed when pretreatment with resveratrol $(\mathrm{P}<0.05)$ (Fig. 4B). It indicated that pretreatment with resveratrol could alleviate the degree of TBI.

Resveratrol attenuated NLRP3 inflammasome signaling activation via enhanced expression of SIRT1. To investigate the mechanism of resveratrol on regulating NLRP3 inflammasome signaling, we further examined the role of SIRT1. We introduced an inhibitor of SIRT1, sirtinol, in conjunction with resveratrol. We found that the SIRT1 expression was
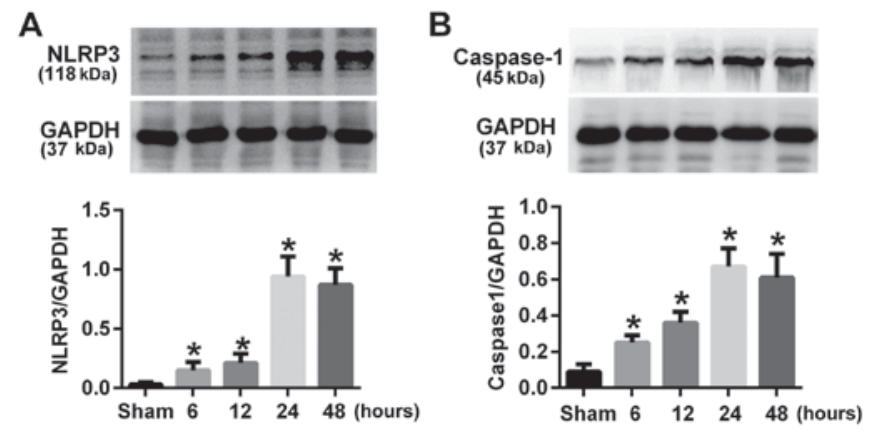

Figure 1. TBI resulted in elevated NLRP3 and caspase-1 expression in rats cerebral cortex. The samples were collected at 6, 12, 24 and $48 \mathrm{~h}$ after TBI. The protein level of (A) NLRP3 and (B) caspase-1 were evaluated by western blot. The values presented are mean \pm SEM ( $n=6$ per group). ${ }^{*} \mathrm{P}<0.05$ compared to the value in Sham group. TBI, traumatic brain injury; NLRP3, NLR family pyrin domain containing 3 .
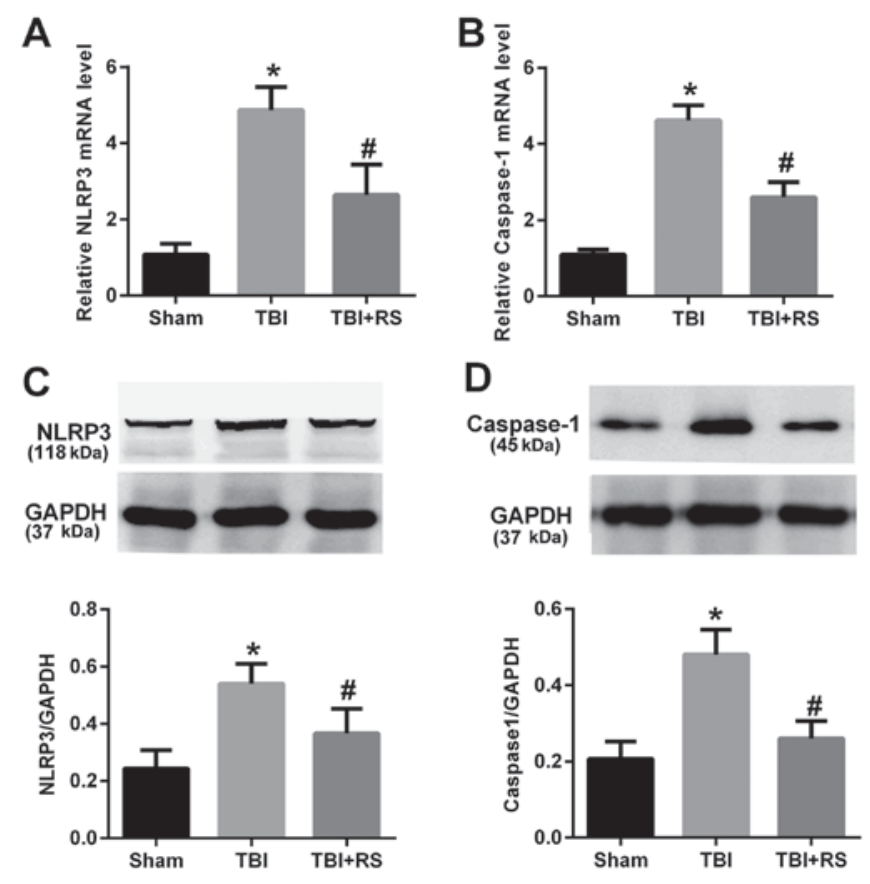

Figure 2. Resveratrol pretreatment reduced the mRNA and protein expression level of NLRP3 and caspase-1 in rats cerebral cortex after TBI. The cerebral cortex samples were collected $24 \mathrm{~h}$ after TBI. The mRNA levels of NLRP3 (A) and caspase-1 (B) were detected by reverse transcription-quantitative polymerase chain reaction. The protein level of (C) NLRP3 and (D) caspase-1 were evaluated by western blot. The values presented are mean \pm standard error mean ( $\mathrm{n}=6$ per group). ${ }^{*} \mathrm{P}<0.05$ compared to the value in Sham group. ${ }^{\#} \mathrm{P}<0.05$ compared to the value in TBI group. TBI, traumatic brain injury; NLRP3, NLR family pyrin domain containing 3; RS, resveratrol.

increased after TBI $(\mathrm{P}<0.05)$, and resveratrol pretreatment further enhanced the expression level of SIRT1 $(\mathrm{P}<0.05)$. When co-administrated with sirtinol and resveratrol, the increased level of SIRT1 in the TBI+RS group was reduced $(\mathrm{P}<0.05)$ (Fig. 5A). Meanwhile, co-administrated with sirtinol and resveratrol reversed the suppressing effect of resveratrol on NLRP3 $(\mathrm{P}<0.05)$ (Fig. 5B). We further examined the level of IL-1 $\beta$ and IL-18. We found co-administration with sirtinol and resveratrol could reverse the reduced level of IL-1 $\beta$ and IL-18 in the TBI+RS group $(\mathrm{P}<0.05)$ (Fig. 5C and D). 

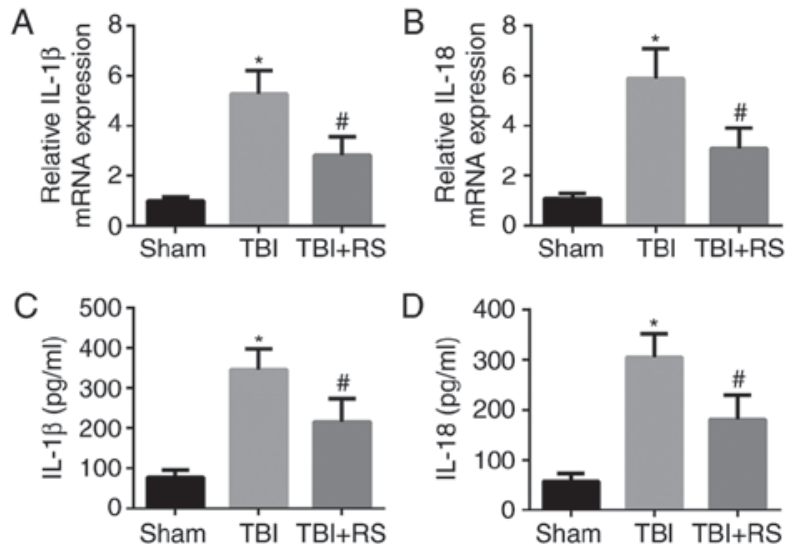

Figure 3. Resveratrol pretreatment decreased the levels of IL-1 $\beta$ and IL-18 in rat cerebral cortex after TBI. The cerebral cortex samples were collected $24 \mathrm{~h}$ after TBI. The mRNA level of IL-1 $\beta$ (A) and IL-18 (B) were determined by reverse transcription-quantitative polymerase chain reaction and normalized to GAPDH. The protein level of IL-1 $\beta$ (C) and IL-18 (D) were measured by ELISA. The values presented are mean \pm standard error mean $(n=6$ per group). ${ }^{*} \mathrm{P}<0.05$ compared to the value in Sham group. ${ }^{\text {}} \mathrm{P}<0.05$ compared to the value in TBI group. IL, interleukin; TBI, traumatic brain injury; RS, resveratrol.
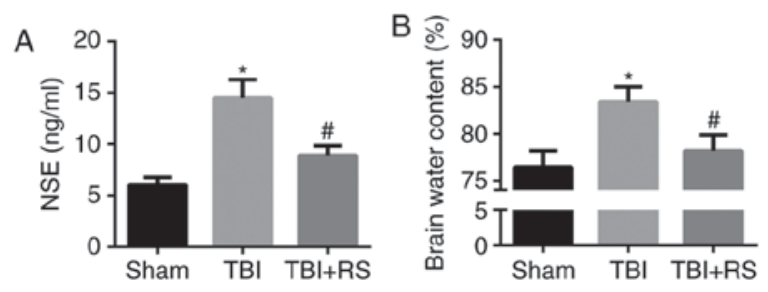

Figure 4. Resveratrol pretreatment decreased the level of serum NSE and BWC of TBI rats. Samples were collected $24 \mathrm{~h}$ after TBI. The level of (A) serum NSE was measured by ELISA and the level of (B) BWC were measured by wet/dry ratio. The values presented are mean \pm standard error mean ( $\mathrm{n}=6$ per group). ${ }^{*} \mathrm{P}<0.05$ compared to the value in Sham group. ${ }^{*} \mathrm{P}<0.05$ compared to the value in TBI group. NSE, neuron-specific enolase; BWC, brain water content; TBI, traumatic brain injury; RS, resveratrol.

Resveratrol pretreatment inhibited the extent of oxidative stress in rats cerebral cortex after TBI via SIRT1. Oxidative stress plays a key role in NLRP3 inflammaoseme activation during ischemia reperfusion injury. To determine whether resveratrol and SIRT1 had an impact on oxidative stress in rats cerebral cortex after TBI, we assessed the changes of endogenous antioxidants SOD, MDA and GSH. We found that the levels of SOD and GSH were remarkably declined, while the level of MDA was significantly elevated in the TBI group compared with the sham group $(\mathrm{P}<0.05)$. When pretreatment with resveratrol, the level of SOD and GSH were elevated while the level of MDA was reduced $(\mathrm{P}<0.05)$. Moreover, sirtinol administration could reverse the inhibiting effect of resveratrol on oxidative stress $(\mathrm{P}<0.05)$ (Fig. 6A-C).

\section{Discussion}

Resveratrol has been demonstrated to exhibit neuroprotective effects in various animal models (17). In the present study, pretreatment with resveratrol had an obviously neuroprotective effect in rats following TBI, as evidenced by reduced
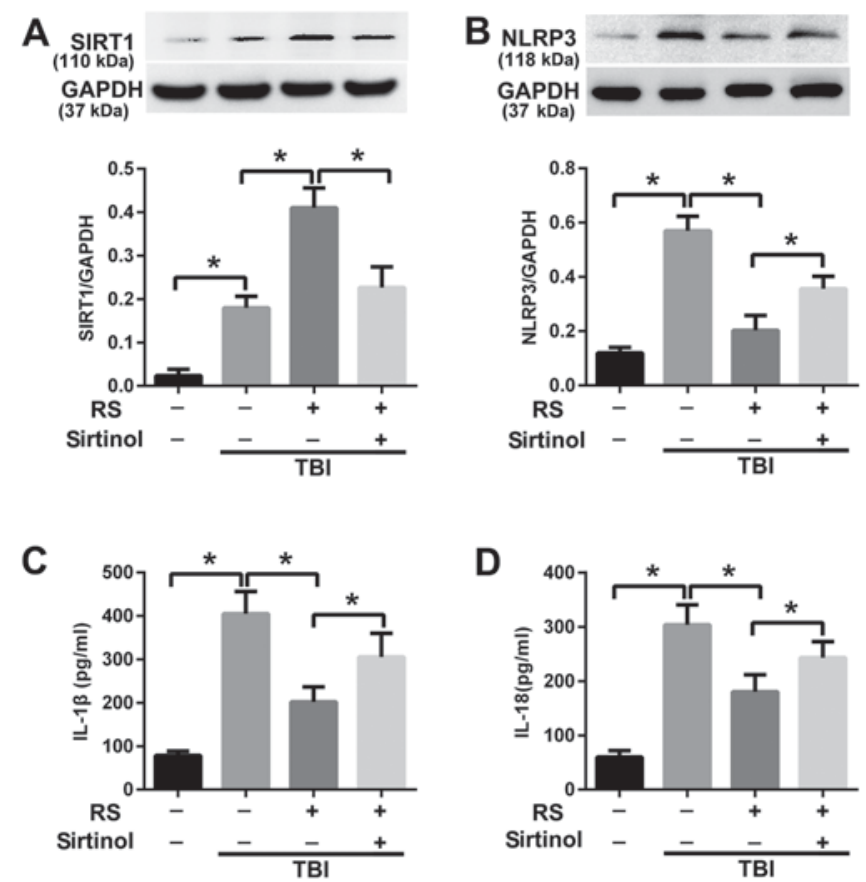

Figure 5. Resveratrol attenuated NLRP3 inflammasome signaling activation via enhanced expression of SIRT1. The cerebral cortex samples were collected $24 \mathrm{~h}$ after TBI. (A and B) Western blot analysis of SIRT1 and NLRP3. (C and D) The protein level of IL-1 $\beta$ and IL-18 measured by ELISA. The values presented are mean \pm standard error mean ( $n=6$ per group). ${ }^{*} \mathrm{P}<0.05$ as indicated. NLRP3, NLR family pyrin domain containing 3 ; SIRT1, sirtuin 1; TBI, traumatic brain injury; IL, interleukin; RS, resveratrol.
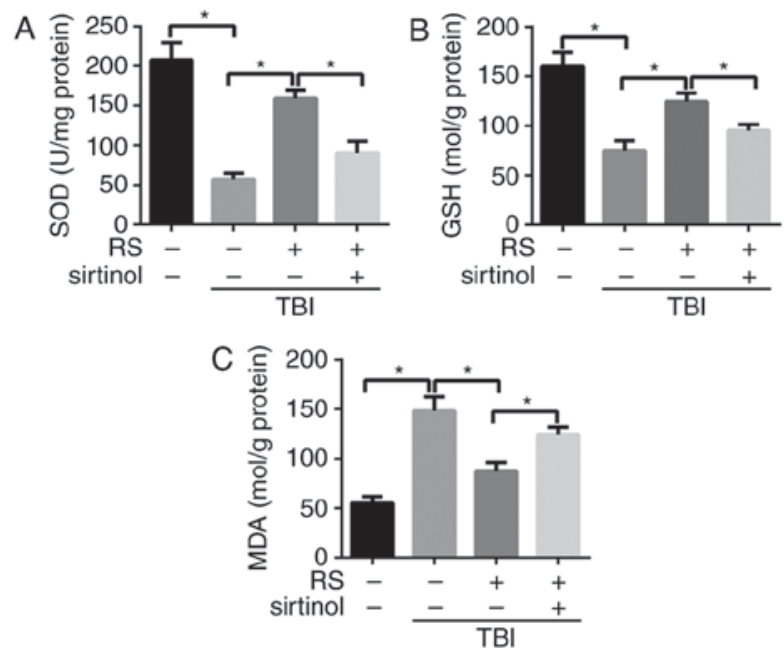

Figure 6. Resveratrol pretreatment inhibited the extent of oxidative stress in rats cerebral cortex after TBI via SIRT1. The cerebral cortex samples were collected $24 \mathrm{~h}$ after TBI. The extent of oxidative stress was represented by SOD activity (A), GSH level (B) and MDA level (C). The values presented are mean \pm standard error mean ( $n=6$ per group). ${ }^{*} \mathrm{P}<0.05$ as indicated. TBI, traumatic brain injury; SOD, superoxide dismutase; GSH, glutathione; MDA, malondialdehyde; SIRT1, sirtuin 1; RS, resveratrol.

serum NSE level and BWC level. Meanwhile, we demonstrated for the first time resveratrol attenuated the inflammatory response and relieved TBI by reducing ROS production and suppressing NLRP3 activation. The effect of resveratrol on NLRP3 inflammasome and ROS production might be SIRT1 dependent. 
There are two stages involved in TBI, including immediate brain damage and secondary injury. The immediate brain damage results in direct neuronal loss and necrotic death, which is followed by a series of injury cascades such as oxidative stress, mitochondrial dysfunction and inflammation (18). Of these injury cascades, inflammatory response accounts for major reasons (19). It is reported that inflammatory responses following various central nervous system injuries, is associated with robust release of cytokines and activation of inflammatory signals, which amplifies the injury (20).

The inflammasome is a multiprotein oligomer consisting of an apoptosis associated speck like protein containing caspase recruitment domain (ASC), a cysteine protease caspase-1 and an NLR protein. Of all inflammasomes, the NLRP3 inflammasome is the most important member. NLRP3 inflammasome is a component of the innate immune system which can promote the maturation of pro-inflammatory cytokines IL-1 $\beta$ and IL-18, thus initiating inflammatory responses (21). In fact, several studies found that the assembly of NLRP3 inflammasome, the activation of caspase- 1 and the release of IL-1 $\beta$ and IL-18 during TBI, indicating NLRP3 was involved in TBI pathology $(8,22,23)$. In the present study, we also observed TBI induced the activation of NLRP3 and its downstream molecule caspase-1. The expression of pro-inflammatory cytokines pro-IL-1 $\beta$ and pro-IL-18 and its maturation pattern IL- $1 \beta$ and IL-18 were also increased in the cerebral cortex of TBI rats.

Literatures indicated that resveratrol had strong neuroprotective effects on central nervous system disorders. It was shown that pretreatment with resveratrol could reduce the infarction volume and improve the neurological score in mouse after middle cerebral artery occlusion (24). Resveratrol was also reported to attenuate TBI induced brain edema and improved spatial cognitive function and neurological impairment in rats $(12,25,26)$. In our study, we used NSE, a specific biomarker closely associated with the damage of neurons, and BWC to represent the degree of brain injury. We found that after TBI, the NSE and BWC were significantly elevated. While pretreatment with resveratrol could remarkably reduce the elevated NSE and brain water content, as well as reduce the activation of NLRP3, caspase- 1 and the release of IL-1 $\beta$ and IL-18. The abovementioned findings indicated that resveratrol might exert a protective effect in TBI by suppressing the NLRP3 inflammasome signaling.

Resveratrol is also known as a potent activator of SIRT1. SIRT1 is a class III deacetylase which involved in multiple physiological or pathological processes such as cell longevity, reduction of oxidative stress, inflammation and metabolism (27-29). It is reported that enhanced expression of SIRT1 had a neuroprotection effect in central nervous system diseases (30). Furthermore, studies had shown that SIRT1 was an endogenous protective molecule in TBI (31), and SIRT1 could negatively regulate NLRP3 in vascular endothelial cells (32). Li et al (32) found that lacking of CD40 could abolishe the up-regulation of NLRP3 expression in LPS-primed ECs and SIRT1 activation could protect against inflammatory response via CD40 both in vivo and in vitro which demonstrated the effect of SIRT1 on NLRP3 inflammasome was partly mediated by CD40. To explore the underlying mechanisms of resveratrol regulating NLRP3 inflammasome in TBI, we used the SIRT1 inhibitor, sirtinol, in our experiment. The concentration of sirtinol we

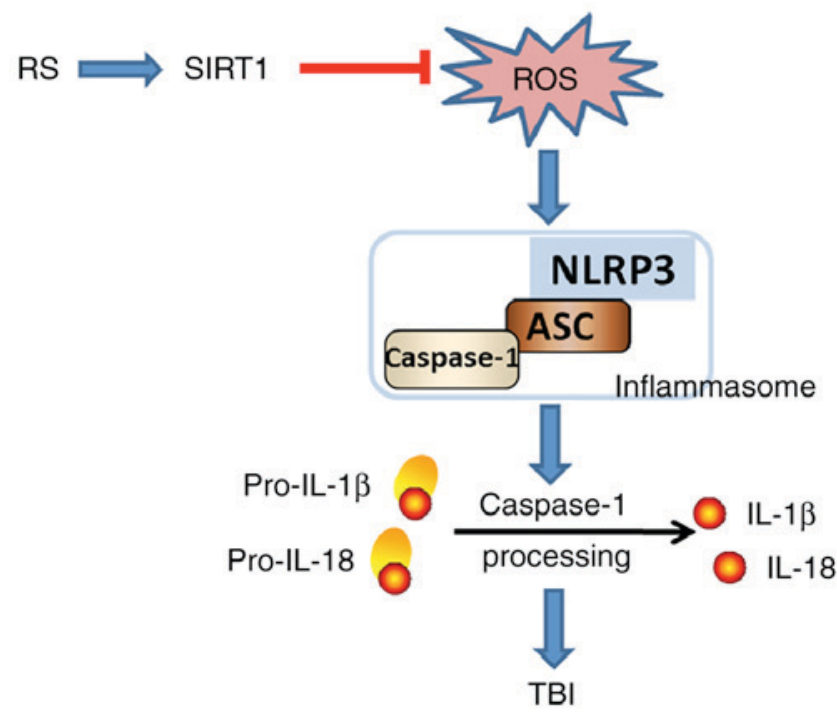

Figure 7. Graphical abstract of resveratrol attenuating inflammatory response and relieving TBI by reducing ROS production and inhibiting NLRP3 activation. The effect of resveratrol on NLRP3 inflammasome and ROS was at least partly SIRT1 dependent. NLRP3, NLR family pyrin domain containing 3; SIRT1, sirtuin 1; RS, resveratrol; ROS, reactive oxygen species; IL, interleukin; ASC, apoptosis associated speck like protein containing caspase recruitment domain; TBI, traumatic brain injury.

used in our experiment was different from the concentration of resveratrol. The concentration of sirtinol was used partly according to previous article (15), and partly attributed to its high potency. We found SIRT1 level was increased in the cerebral cortex after TBI, while pretreatment with resveratrol resulted in a higher level of SIRT1. When co-administrated with sirtinol, we observed downregulated level of SIRT1. The blockade of SIRT1 activation by sirtinol was associated with enhanced NLRP3 inflammaosme activation in TBI. These results indicated the mechanism of resveratrol suppressing NLRP3 inflammaosme was SIRT1 dependent.

Various exogenous and endogenous molecular patterns can activate NLRP3 inflammasome. Of them, reactive oxygen species (ROS) have been regarded as an important NLRP3 inflammasome activator in various diseases, such as cardiac ischemia/reperfusion injury (33) and sepsis-induced acute lung injury (34). Meanwhile, SIRT1 is an important oxidative stress regulator (29). It is reported that TBI resulted in rapid ROS production and oxidative damage to brain cellular components leading to neuronal dysfunction and cell death (35). ROS is constantly generated and eliminated in the biological homoeostasis, which is controlled by endogenous antioxidant system, including antioxidants such as GSH and SOD. SOD and GSH are ROS scavengers and can eliminate the generated ROS in vivo (36). MDA is a production of oxidative stress of lipid peroxidation, and it is generated when polyunsaturated lipids are oxidized by ROS. ROS production can be represented by the level of SOD, GSH and MDA (37). To determine whether resveratrol could affect the ROS level through SIRT1 in cerebral cortex after TBI, we conducted further experiments. In the present study, we found that after TBI, the level of SOD and GSH were significantly reduced while the level of MDA was remarkably increased, indicating plenty of ROS production. When pretreatment with resveratrol, the level of SOD and GSH 
were elevated while the level of MDA was reduced, indicating resveratrol could decrease the production of ROS. Moreover, co-administrated with sirtinol remarkably reversed the effect of resveratrol on SOD, GSH and MDA. These results indicated that the effect of resveratrol on suppressing NLRP3 inflammasome activation and attenuating the cerebral cortex injury after TBI might be related to the reduced ROS production, and this effect of resveratrol on NLRP3 inflammasome and ROS production might be SIRT1 dependent (Fig. 7).

In conclusion, our data demonstrated that TBI could activate NLRP3 inflammasome, thus promoting the release of proinflammatory cytokines, IL-1 $\beta$ and IL-18, and amplifying brain injury. Resveratrol might attenuate the inflammatory response and relieve TBI by reducing ROS production and inhibiting NLRP3 activation, which prevented the over-release of pro-inflammatory cytokines. The effect of resveratrol on NLRP3 inflammasome and ROS production might be SIRT1 dependent. The present study enriched the anti-inflammatory mechanisms of resveratrol and enhanced the therapeutic potential of resveratrol for the intervention of TBI.

\section{References}

1. Dong HJ, Shang CZ, Li G, Niu Q, Luo YC, Yang Y, Meng HP, Yin HJ, Zhang HX, Zhao ML and Lin L: The distribution of transplanted umbilical cord mesenchymal stem cells in large blood vessel of rats with traumatic brain injury. J Craniofac Surg, Mar 29, 2017 (Epub ahead of print).

2. Kumar A, Stoica BA, Loane DJ, Yang M, Abulwerdi G, Khan N, Kumar A, Thom SR and Faden AI: Microglial-derived microparticles mediate neuroinflammation after traumatic brain injury. J Neuroinflammation 14: 47, 2017.

3. Jin R, Shen M, Yu L, Wang X and Lin X: Adipose-derived stem cells suppress inflammation induced by IL-1 $\beta$ through down-regulation of P2X7R mediated by miR-373 in chondrocytes of osteoarthritis. Mol Cells 40: 222-229, 2017.

4. Song L, Pei L, Yao S, Wu Y and Shang Y: NLRP3 inflammasome in neurological diseases, from functions to therapies. Front Cell Neurosci 11: 63, 2017.

5. Minutoli L, Puzzolo D, Rinaldi M, Irrera N, Marini H, Arcoraci V, Bitto A, Crea G, Pisani A, Squadrito F, et al: ROS-mediated NLRP3 inflammasome activation in brain, heart, kidney, and testis ischemia/reperfusion injury. Oxid Med Cell Longev 2016: 2183026, 2016

6. Tan MS, Yu JT, Jiang T, Zhu XC and Tan L: The NLRP3 inflammasome in Alzheimer's disease. Mol Neurobiol 48: 875-882, 2013

7. Li L and Liu Y: Aging-related gene signature regulated by Nlrp3 predicts glioma progression. Am J Cancer Res 5: 442-449, 2014.

8. Liu HD, Li W, Chen ZR, Hu YC, Zhang DD, Shen W, Zhou ML, Zhu L and Hang CH: Expression of the NLRP3 inflammasome in cerebral cortex after traumatic brain injury in a rat model. Neurochem Res 38: 2072-2083, 2013.

9. Jardim FR, de Rossi FT, Nascimento MX, da Silva Barros RG, Borges PA, Prescilio IC and de Oliveira MR: Resveratrol and brain mitochondria: A review. Mol Neurobiol: Mar 10, 2017 (Epub ahead of print).

10. Abdel-Aleem GA, Khaleel EF, Mostafa DG and Elberier LK: Neuroprotective effect of resveratrol against brain ischemia reperfusion injury in rats entails reduction of DJ-1 protein expression and activation of PI3K/Akt/GSK3b survival pathway. Arch Physiol Biochem 122: 200-213, 2016.

11. Wan D, Zhou Y, Wang K, Hou Y, Hou R and Ye X: Resveratrol provides neuroprotection by inhibiting phosphodiesterases and regulating the cAMP/AMPK/SIRT1 pathway after stroke in rats. Brain Res Bull 121: 255-262, 2016.

12. Feng Y, Cui Y, Gao JL, Li MH, Li R, Jiang XH, Tian YX, Wang KJ, Cui CM and Cui JZ: Resveratrol attenuates neuronal autophagy and inflammatory injury by inhibiting the TLR4/NF- $\mathrm{BB}$ signaling pathway in experimental traumatic brain injury. Int J Mol Med 37: 921-930, 2016.

13. Jiang L, Zhang L, Kang K, Fei D, Gong R, Cao Y, Pan S, Zhao M and Zhao M: Resveratrol ameliorates LPS-induced acute lung injury via NLRP3 inflammasome modulation. Biomed Pharmacother 84: 130-138, 2016.
14. Su Q, Pu H and $\mathrm{Hu} \mathrm{C}$ : Neuroprotection by combination of resveratrol and enriched environment against ischemic brain injury in rats. Neurol Res 38: 60-68, 2016.

15. Yang H, Gu ZT, Li L, Maegele M, Zhou BY, Li F, Zhao M and Zhao KS: SIRT1 plays a neuroprotective role in traumatic brain injury in rats via inhibiting the p38 MAPK pathway. Acta Pharmacol Sin 38: 168-181, 2017.

16. Wang K, Zhang L, Rao W, Su N, Hui H, Wang L, Peng C, Tu Y, Zhang S and Fei Z: Neuroprotective effects of crocin against traumatic brain injury in mice: Involvement of notch signaling pathway. Neurosci Lett 591: 53-58, 2015.

17. Lopez MS, Dempsey RJ and Vemuganti R: Resveratrol neuroprotection in stroke and traumatic CNS injury. Neurochem Int 89: 75-82, 2015.

18. Gardner AJ, Shih SL, Adamov EV and Zafonte RD: Research frontiers in traumatic brain injury: Defining the injury. Phys Med Rehabil Clin N Am 28: 413-431, 2017.

19. Naghibi T, Mohajeri M and Dobakhti F: Inflammation and outcome in traumatic brain injury: Does gender effect on survival and prognosis? J Clin Diagn Res 11: PC06-PC09, 2017.

20. Russo MV and McGavern DB: Inflammatory neuroprotection following traumatic brain injury. Science 353: 783-785, 2016.

21. Pennisi M,Crupi R, Di Paola R, Ontario ML, Bella R, Calabrese EJ, Crea R, Cuzzocrea S and Calabrese V: Inflammasomes, hormesis, and antioxidants in neuroinflammation: Role of NRLP3 in Alzheimer disease. J Neurosci Res 95: 1360-1372, 2017.

22. Wallisch JS, Simon DW, Bayir H, Bell MJ, Kochanek PM and Clark RSB: Cerebrospinal fluid NLRP3 is increased after severe traumatic brain injury in infants and children. Neurocrit Care 27: 44-50, 2017.

23. Lin C, Chao H, Li Z, Xu X, Liu Y, Bao Z, Hou L, Liu Y, Wang X, You Y, et al: Omega-3 fatty acids regulate NLRP3 inflammasome activation and prevent behavior deficits after traumatic brain injury. Exp Neurol 290: 115-122, 2017.

24. Shah FA, Gim SA, Kim MO and Koh PO: Proteomic identification of proteins differentially expressed in response to resveratrol treatment in middle cerebral artery occlusion stroke model. J Vet Med Sci 76: 1367-1374, 2014.

25. Lin CJ, Chen TH, Yang LY and Shih CM: Resveratrol protects astrocytes against traumatic brain injury through inhibiting apoptotic and autophagic cell death. Cell Death Dis 5: e1147, 2014.

26. Gatson JW, Liu MM, Abdelfattah K, Wigginton JG, Smith S, Wolf S and Minei JP: Resveratrol decreases inflammation in the brain of mice with mild traumatic brain injury. J Trauma Acute Care Surg 74: 470-475, 2013.

27. Matsushima S and Sadoshima J: The role of sirtuins in cardiac disease. Am J Physiol Heart Circ Physiol 309: H1375-H1389, 2015.

28. Kleszcz R, Paluszczak J and Baer-Dubowska W: Targeting aberrant cancer metabolism-The role of sirtuins. Pharmacol Rep 67: 1068-1080, 2015.

29. Tang BL: Sirt1 and the mitochondria. Mol Cells 39: 87-95, 2016.

30. Martin A, Tegla CA, Cudrici CD, Kruszewski AM, Azimzadeh P, Boodhoo D, Mekala AP, Rus V and Rus H: Role of SIRT1 in autoimmune demyelination and neurodegeneration. Immunol Res 61: 187-197, 2015.

31. Zhao Y, Luo P, Guo Q, Li S, Zhang L, Zhao M, Xu H, Yang Y, Poon W and Fei Z: Interactions between SIRT1 and MAPK/ERK regulate neuronal apoptosis induced by traumatic brain injury in vitro and in vivo. Exp Neurol 237: 489-498, 2012.

32. Li Y, Yang X, He Y, Wang W, Zhang J, Zhang W, Jing T, Wang B and Lin R: Negative regulation of NLRP3 inflammasome by SIRT1 in vascular endothelial cells. Immunobiology 222: 552-561, 2017.

33. Liu Y, Lian K, Zhang L, Wang R, Yi F, Gao C, Xin C, Zhu D, Li Y, Yan W, et al: TXNIP mediates NLRP3 inflammasome activation in cardiac microvascular endothelial cells as a novel mechanism in myocardial ischemia/reperfusion injury. Basic Res Cardiol 109: 415, 2014.

34. Yin N, Peng Z, Li B, Xia J, Wang Z, Yuan J, Fang L and Lu X: Isoflurane attenuates lipopolysaccharide-induced acute lung injury by inhibiting ROS-mediated NLRP3 inflammasome activation. Am J Transl Res 8: 2033-2046, 2016.

35. Butterfield DA and Reed TT: Lipid peroxidation and tyrosine nitration in traumatic brain injury: Insights into secondary injury from redox proteomics. Proteomics Clin Appl 10: 1191-1204, 2016.

36. Rodriguez-Rodriguez A, Egea-Guerrero JJ, Murillo-Cabezas F and Carrillo-Vico A: Oxidative stress in traumatic brain injury. Curr Med Chem 21: 1201-1211, 2014.

37. Czerska M, Mikołajewska K, Zieliński M, Gromadzińska J and Wasowicz W: Today's oxidative stress markers. Med Pr 66: 393-405, 2015. 